\title{
Complex Permeability Measurements in a Nanocrystalline Toroidal Core
}

\author{
Hajer Lagha ${ }^{1,2}$, Hafedh Belmabrouk ${ }^{1,3^{*}}$, Hervé Chazal ${ }^{2}$ \\ ${ }^{1}$ Laboratory of Electronics and Microelectronics, Faculty of Sciences of Monastir, \\ University of Monastir, Tunisia \\ ${ }^{2}$ Electrical Engineering Laboratory (G2Elab), University of Grenoble Alpes, France \\ ${ }^{3}$ Department of Physics, College of Science Al-Zulfi, Majmaah University, KSA \\ * Corresponding author email: ha.belmabrouk@mu.edu.sa; hafedh.belmabrouk@fsm.rnu.tn
}

Received: 24 April 2016 / Revised: 25 May 2016 / Accepted: 03 June 2016 / Published: 15 June 2016

\begin{abstract}
In the design of power electronic converters, the choice of the suitable magnetic alloys and the measurement of the variations of their magnetic properties versus the temperature are fundamental issues. A theoretical model based on Neel theory that takes into account wall displacement and coherent rotation is elaborated to derive the expression of the complex permeability versus the frequency. The static and the dynamic behaviour has been considered in this model. The dependence of temperature is implicit in this model, i.e. the parameters involved in the expression of the complex permeability may depend on the temperature. An impedance analyser has been used to measure the open circuit and short circuit complex impedances of a toroidal core versus the frequency at several temperatures ranging from $20^{\circ} \mathrm{C}$ to $180^{\circ} \mathrm{C}$. The complex permeability of the material is then deduced. Finally, the theoretical model is used to identify the domain susceptibility and the anisotropy energy of the materials. The effect of temperature on these parameters is analyzed. The results obtained are valuable for the design of power electronic converters from the magnetic and thermal viewpoints, which prove the need for the combination of heat transfer and magnetic mechanisms at the design.
\end{abstract}

Keywords: Anisotropy energy, Complex permeability, Domain susceptibility, Nanocrystalline material, Neel model, Temperature dependence

\section{Introduction}

Power electronic converters are widely used in many industrial applications. Their main functions are voltage conversion and regulation. Various safety standards of power electronic converters should also be satisfied. Considerable architecture is available, and the classification of power conversion systems depends on whether the input and output are alternating current or direct current or voltage [1-3]. To improve the efficiency of the magnetic components such as power converters and to avoid the need of sophisticated cooling systems, it is necessary to limit the heat generation in the magnetic components. In the design of a power converter, it is important to choose the appropriate alloy from a magnetic point of view but it is also important to take into account the thermal constraints due to heat generation and the dependence of the magnetic properties on the temperature [4-9].

In this paper, the complex permeability of a toroidal core of nanocrystalline FeNbCuSiB alloys is indirectly measured versus the frequency and at different temperatures ranging from $20^{\circ} \mathrm{C}$ to $180^{\circ} \mathrm{C}$. A theoretical model is used to identify the domain susceptibility and the anisotropy energy from the complex permeability 
measurements. The dependence of these parameters versus the temperature is given.

\section{Mathematical Formulation}

Nanocrystalline ribbons are used to perform the toroidal core simulation of a converter. An electromagnetic propagation wave model is used to evaluate the complex permeability $\tilde{\mu}=$ $\mu_{0}\left(\mu^{\prime}-j \mu^{\prime \prime}\right)$ (where $\mu_{0}$ is the vacuum permeability; $\mu^{\prime}$ and $\mu^{\prime \prime}$ are real and imaginary parts of the relative complex permeability). To take into account the non-homogeneity of the magnetic material, the domain walls description has been used [7]. We adopt the well-known Neel model. The following assumptions are made:

* The external magnetic field exciting the material is sinusoidal with an angular frequency $\omega$;

* The amplitude of external magnetic field $H_{0}$ is small;

* The ferromagnetic material behaves as a linear media. Therefore, it is possible to use the concept of permeability to describe its macroscopic behaviour;

* The material is anisotropic. The magnetic field $\vec{H}$ and the magnetization field $\vec{M}$ are not collinear. The angle between these two vectors is designed by $\alpha$;

* The ferromagnetic film has the structure of a two-dimensional $180^{\circ}$ Bloch-type domain wall (figure 1). The distance $d$ between two consecutive domains is supposed to be constant. The thickness of the slab is designed by $\delta$;

* Two mechanisms contribute to the magnetization, namely the wall displacement and coherent rotations;

* The direction of the magnetization field $\vec{M}$ deviates with an angle $\theta$ under the effect of the magnetic field;

* The component of the magnetic field perpendicular to the magnetization field does not apply a force on the domain walls. In other words, it does not contribute to the wall displacement.

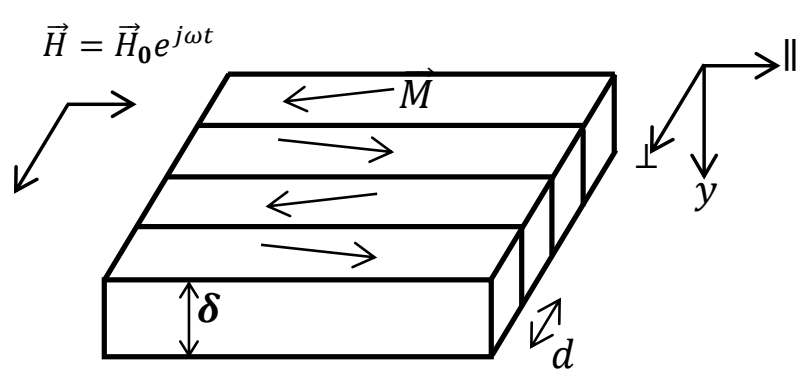

Figure 1: Wall domains in a ferromagnetic material

The magnetic field $\vec{H}$ may be decomposed in a basis whose directions are parallel and perpendicular to the magnetization field $\vec{M}$ (figure 2).

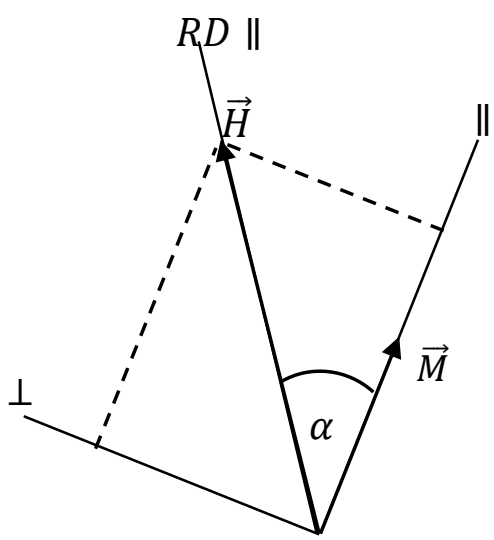

Figure 2: Decomposition of the magnetic field into parallel and perpendicular parts

The susceptibility is given by the matrix $(\chi)$ :

$$
\left(\begin{array}{l}
\delta M_{\|} \\
\delta M_{\perp}
\end{array}\right)=\left(\begin{array}{ll}
\chi_{11} & \chi_{12} \\
\chi_{21} & \chi_{22}
\end{array}\right)\left(\begin{array}{l}
\delta H_{\|} \\
\delta H_{\perp}
\end{array}\right)
$$

It is also useful to decompose the vectors on the axis parallel and perpendicular to the rolling direction (RD).

It is straightforward to obtain the susceptibility matrix $\chi_{R D}$ in this basis from the matrix $(\chi)$ and the angle $\alpha$. It is also worthwhile to relate each term to the effect of wall displacement or coherent rotations. For instance, we have:

$$
\chi_{11}=\left(\frac{\delta M_{\|}}{\delta H_{\|}}\right)_{\delta H_{\perp}=0}
$$

The above term is equal to that obtained by the wall displacement model [7]. Regarding the third term, we obtain $\chi_{21}=0$ since the component $H_{\|}$cannot provoke any magnetization variation 
in the perpendicular direction. The terms $\chi_{12}$ and $\chi_{22}$ are due to coherent rotations:

Magnetic field diffusion equation is written for the components $H_{\|}$and $H_{\perp}$. To resolve these equations, one should add the boundary conditions. The general solution may be obtained by using a Fourier series decomposition. A laborious technique has been detailed by [7]. The dynamic permeability $\mu_{\perp}$ in the direction perpendicular to the magnetization direction is given by:

$$
\begin{gathered}
\mu_{\perp}(\omega)=\frac{\tanh (X)}{X} \mu_{\perp} \\
X=\frac{\delta}{2} \sqrt{j \omega \sigma \mu_{0} \mu_{\perp}}
\end{gathered}
$$

$\sigma$ is the electrical conductivity of the material and $\mu_{\perp}$ is scalar real permeability. It should be noted that $\mu_{\perp}$ depends on the anisotropy energy $\mathrm{Ku}$ [7]. The dynamic permeability $\mu_{R D \|}$ in the direction parallel to the rolling direction is given by:

$$
\begin{aligned}
\mu_{R D \|}(\omega)=4 \mu_{0} & \frac{\chi_{w}}{d} A^{2} \cos ^{2}(\alpha) \\
& +\mu_{0} \mu_{\perp}(\omega) \sin ^{2}(\alpha)
\end{aligned}
$$

$\chi_{w}$ is the domain susceptibility of the material. It is related to the initial static susceptibility of the material. The parameter $A$ depends on the coefficients of the Fourier series decomposition. Its expression is available in [7]. It is also worthwhile to write the relation for the susceptibility at low frequencies. The above relations allow to compute the permeability $\mu_{R D \|}(\omega)$ and the permeability $\mu_{\perp}(\omega)$ versus the frequency when the parameters $\chi_{w}, d, \mu_{\perp}, \alpha, \sigma$ and $\delta$ are known. On the other hand, the measurement of the real part and imaginary part of the permeability $\mu_{R D \|}(\omega)$ leads to the identification of these parameters using a nonlinear algorithm. It should be noted that the temperature does not appear explicitly in the above model. However, the parameters involved may depend on the temperature. For this reason, the experiment is done at different temperatures to analyse the dependence of the identified parameters versus the temperature.

\section{Experimental Device}

The permeability of the material will be measured indirectly. Indeed, we will perform some impedance measurements from which we will deduce the permeability as shown hereafter. A toroidal core of $20 \mu \mathrm{m}$ nanocrystalline ribbons will be used to measure indirectly the permeability of the material. The numbers of turns of the primary and secondary windings are respectively noted $N_{p}$ and $N_{s}$. The geometrical features of the toroidal core to be tested are summarized in table 1. Primary and secondary windings are wound in diametrical commonmode chokes (CMC) topology to carry out mutual impedance measurement.

Several techniques are available to measure the impedance. In this work, we used the Agilent 4294A impedance analyser. This device combines 4-terminal pair measurement method into an auto-balanced bridge circuit [10]. The Agilent analyser indicates modulus and argument of each impedance measurement. An open-short compensation has been performed out before starting the measurements process to avoid wiring impact. The Agilent analyser allows many kinds of impedance measurement such as opencircuit and short-circuit impedances. More details on the device are available in the technical documentation of Agilent [10]. Measurements are made at different temperatures from $20^{\circ} \mathrm{C}$ to 180 ${ }^{\circ} \mathrm{C}$ using thermal chamber and at different frequencies from $40 \mathrm{~Hz}$ to $110 \mathrm{MHz}$. These measurements are made by using an experimental test bench at each temperature. As shown in figure 3 , the test bench consists of a PC (data acquisition module), the Agilent 4294A analyser with test fixture 1604E (40 Hz to $110 \mathrm{MHz}$ ) and the thermal chamber (to control the temperature).

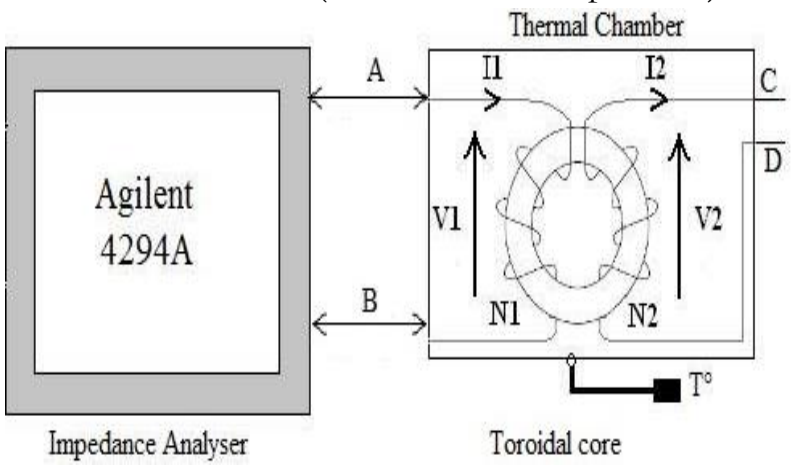

Figure 3: Experimental set-up 
Lagha et al., J. Mod. Mater.; Vol. 1, Issue 1, pp: 2-8, 2016

To deduce the complex permeability at given frequency and temperature many impedance measurements are performed. The experimental protocol is well explained in the provider documentation. As shown in figure 3 , the toroidal core possesses 4 -linked ports A, B, C and D. The analyser allows the measurements of the 4 following impedances $Z_{o}, Z_{o}^{\prime}, Z_{s}$ and $Z_{s}^{\prime}: Z_{o}$ is the impedance seen from the primary when secondary winding is open circuited, $Z_{S}$ is the impedance seen from the primary when secondary winding is short circuited, $Z_{o}^{\prime}$ is the impedance seen from the secondary when primary winding is open circuited and $Z_{S}^{\prime}$ is the impedance seen from the secondary when primary winding is short circuited. To qualify measurement reliability, the confidence factor is introduced [11]. This factor is a useful evaluator and is defined by:

$$
C F=\left|\frac{Z_{o} Z_{s}^{\prime}}{Z_{o}^{\prime} Z_{s}}\right|
$$

This quantity should be equal to 1 whatever the frequency. Any deviation from the unity is a sign of measurement errors.

\section{Results and Discussion}

The approach adopted in this work is divided into three steps:

* First, we will present the experimental results related to the variations of the complex impedances $Z_{o}, Z_{o}^{\prime}, Z_{s}$ and $Z_{s}^{\prime}$ versus the frequency and at several temperatures ranging from $20{ }^{\circ} \mathrm{C}$ to $180^{\circ} \mathrm{C}$. From these measurements, we obtain the magnetizing inductance $Z_{p}$ of the equivalent circuit;

* In the second step, we calculate the complex permeability at different temperatures using the data related to the magnetizing inductance $Z_{p}$ and some geometrical and physical parameters of the device;

* Finally, we use the experimental data and the semi-analytical model to identify fit parameters at each temperature. Variations of these parameters versus temperature are analysed.

\subsection{Impedance Measurement}

The impedances $Z_{o}, Z_{o}^{\prime}, Z_{s}$ and $Z_{s}^{\prime}$ versus the frequency $f$ at several temperatures. Figure 4 and figure 5 exhibit the modulus and the argument of the impedances $Z_{o}, Z_{o}^{\prime}, Z_{s}$ and $Z_{S}^{\prime}$ versus the frequency $f$ at $\mathrm{T}=40{ }^{\circ} \mathrm{C}$.

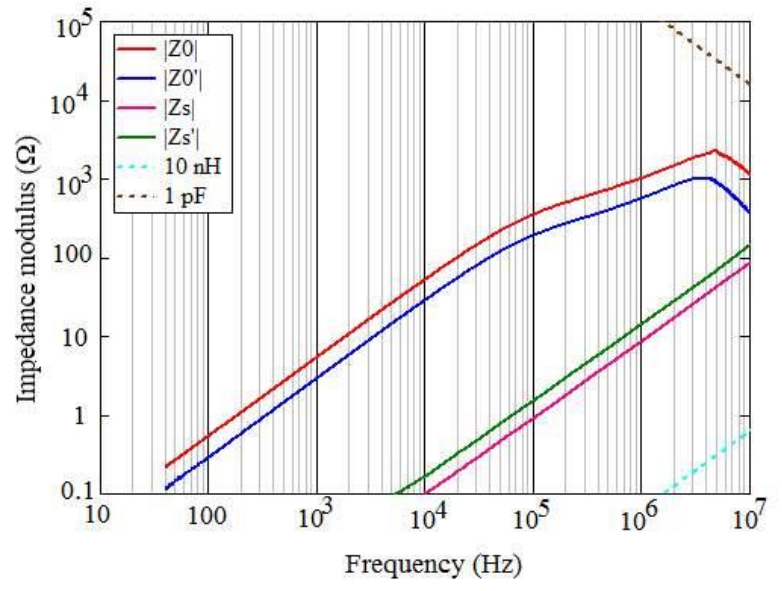

Figure 4: Open and short circuit impedance modulus at $40{ }^{\circ} \mathrm{C}$

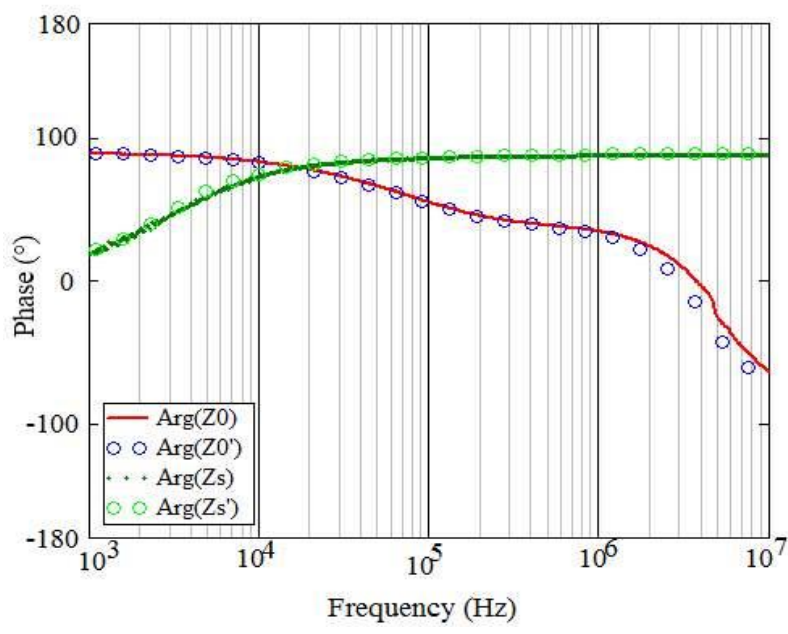

Figure 5: Open and short circuit impedance arguments at $40^{\circ} \mathrm{C}$

The doted lines in figure 4 correspond to the impedance of $10 \mathrm{nH}$ inductor and a $1 \mathrm{pF}$ capacitor, giving a rough idea of resolution limits of equipment used. We notice that the impedances vary in a large range when the frequency increases. For large frequencies, the open circuit impedance arguments become negative. This means that the impedances change from inductive to capacitive behaviour. However, the short circuit impedance arguments tend to the value $\pi / 2$ for high frequencies. 


\subsection{Complex Permeability}

Figure 6 presents the equivalent circuit of the toroidal core in the framework of the lumped element model. Two serial resistances $r_{1}$ and $r_{2}, a$ magnetizing inductance $L_{p}$ and a leakage inductance $L_{f}$ are required to describe this equivalent circuit.

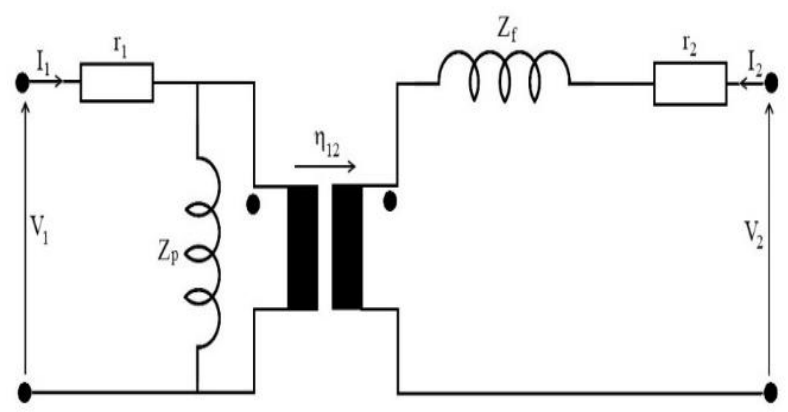

Figure 6: Two windings transformer

It is obvious that the resistances $r_{1}$ and $r_{2}$ can be obtained respectively from $Z_{o}$ and $Z_{o}^{\prime}$ curves at low frequencies. It is straightforward to obtain that the impedance related to the magnetizing inductance is given by:

$$
Z_{p}(\omega)=\operatorname{Im}\left(Z_{o}(\omega)-r_{1}\right)
$$

We can then obtain the values of $Z_{p}$ versus the frequency and at several temperatures. The relative dynamic permeability of the toroidal core made from the nanocrystalline $\mathrm{FeNbCuSiB}$ reads [9]:

$$
\mu(\omega)=\frac{Z_{p}(\omega) L_{m}}{\omega N_{p} N_{s} A_{E} \mu_{0}}
$$

The geometrical parameters involved in Equation (8) are presented in table 1 . This equation enables us to plot the complex permeability versus the frequency at different values of the operating temperature.

Table 1: Geometric parameters of the toroidal core

\begin{tabular}{|l|c|}
\hline Inner diameter $(\mathrm{mm})$ & 10.7 \\
\hline Outer diameter $(\mathrm{mm})$ & 17 \\
\hline Average length $L_{m}(\mathrm{~mm})$ & 43.5 \\
\hline Height $(\mathrm{mm})$ & 6.2 \\
\hline Cross section area $A_{E}\left(\mathrm{~mm}^{2}\right)$ & 15.6 \\
\hline Primary number of turns $N_{p}$ & 11 \\
\hline Secondary number of turns $N_{s}$ & 7 \\
\hline
\end{tabular}

Figure 7 and figure 8 depict the real part $\mu^{\prime}$ and the imaginary part $\mu^{\prime \prime}$ of the complex permeability versus the frequency for two values of the temperature.

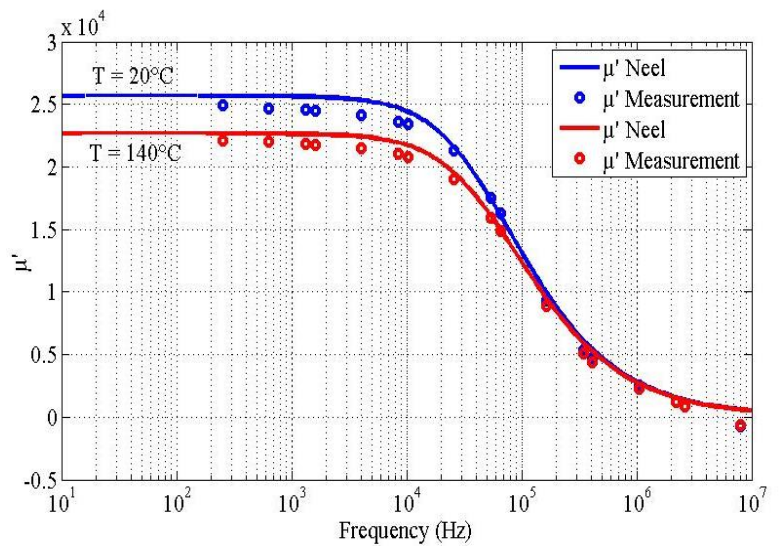

Figure 7: Real part of the complex permeability versus the frequency at $T=20^{\circ} \mathrm{C}$ and $\mathrm{T}=140^{\circ} \mathrm{C}$

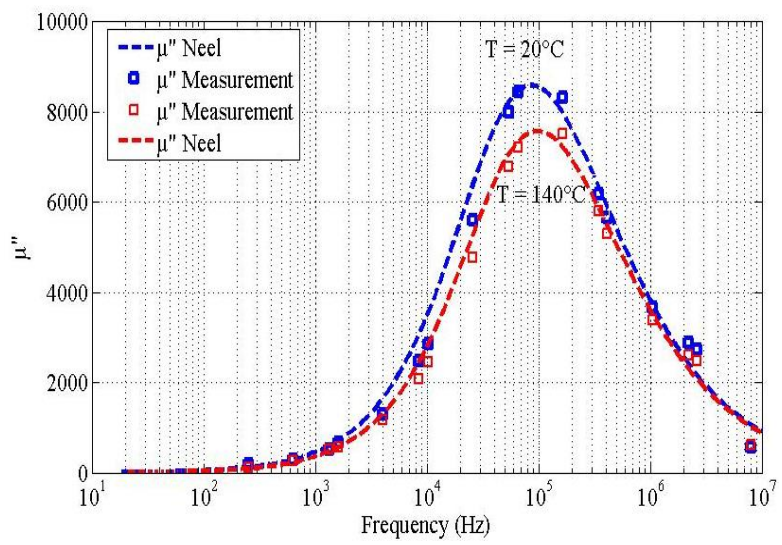

Figure 8: Imaginary part of the complex permeability versus the frequency at $T=20^{\circ} \mathrm{C}$ and $T=140{ }^{\circ} \mathrm{C}$

When the frequency $f$ increases, the real part of the complex permeability remains almost constant when $f$ is less than $10 \mathrm{kHz}$. Then $\mu^{\prime}$ decreases significantly. However, the imaginary part $\mu^{\prime \prime}$ exhibits a maximum for a frequency about $70 \mathrm{kHz}$. On the other hand, when the temperature increases, both the real part $\mu^{\prime}$ and the imaginary part $\mu^{\prime \prime}$ decrease. Since $\mu^{\prime \prime}$ is mainly due to the electrical conductivity $\sigma$, therefore the temperature does not affect the electrical conductivity at large frequencies. We may conclude that the temperature increase provokes a modification of the magnetic behaviour of the alloys. For this reason, the simulation and design of a power converter require not only the dependence of $\mu^{\prime}$ and $\mu^{\prime \prime}$ versus the frequency but also the thermal effect. 
Lagha et al., J. Mod. Mater.; Vol. 1, Issue 1, pp: 2-8, 2016

It is expected that the heat transfer inherent to the converter operation has a significant effect on its performance. Therefore, it is important to take into account the magnetic and thermal proprieties of the material. Compact models should involve magnetic part as well as a thermal part [11]. In this study, the magnetic and thermal effects have been treated independently since the temperature has been considered as a parameter that can influence magnetic properties. Actually, the two mechanisms are coupled and we have to resolve heat transfer equation to obtain the operating temperature. The source term in this equation is obtained by magnetic fields [11-12].

\subsection{Parameter Identification}

As mentioned in section 2, the theoretical model involves some parameters which depend on frequency and temperature. The fit of experimental data using the theoretical model allows the identification of these quantities and the validation of the model. To simplify the identification procedure, we will start by analysing the behaviour of the permeability at low frequencies. This step allows us to identify the anisotropy energy $\mathrm{Ku}$ and the angle between the magnetic field and the magnetization $\alpha$. In the second step, we use all the permeability curve to identify unambiguously the average domain thickness $d$ and the domain susceptibility $\chi_{w}$. An iterative algorithm has been elaborated and validated to perform this task. The algorithm is also based on minimization of errors using least squares method.

Figure 7 and figure 8 show the comparison of the real part $\mu^{\prime}$ and the imaginary part $\mu^{\prime \prime}$ of the complex permeability at two values of temperature. It is clear that the theoretical model fits well the experimental data. This result validates the identification process.

Figure 9 exhibits the variation of anisotropy energy versus the temperature. It is clear that the anisotropy depends strongly on the temperature. It declines from $1200 \mathrm{~J} / \mathrm{m}^{3}$ at $20{ }^{\circ} \mathrm{C}$ to 400 $\mathrm{J} / \mathrm{m}^{3}$ at $180^{\circ} \mathrm{C}$. As the temperature approaches the Curie point, the material becomes more and more isotropic. A similar behaviour has been observed for some materials such as magnetite
$\left(\mathrm{Fe}_{3} \mathrm{O}_{4}\right)$ (Curie point $\left.280 \mathrm{~K}\right)$ [13] and nanoparticles $\left(\mathrm{MnFe}_{2} \mathrm{O}_{4}\right)$ (Curie point $125 \mathrm{~K}$ ) [14]. The obtained result proves that thermal heating of a converter has an important effect on the anisotropy energy. This finding suggests that heat transfer is expected to impact strongly magnetic performance of a converter and it is necessary to develop new models that are able to deal simultaneously with magnetic and thermal mechanisms.

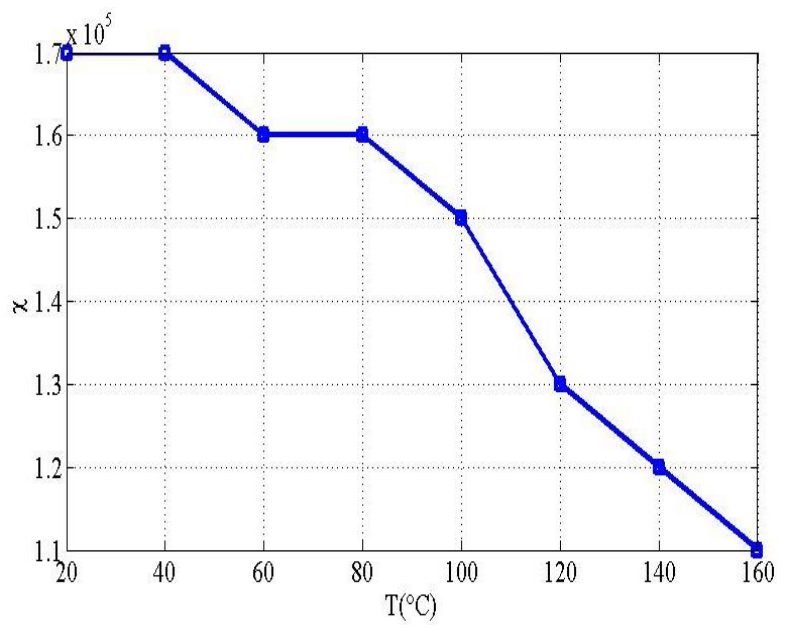

Figure 9: Variations of the domain susceptibility with the temperature.

Figure 10 shows the temperature dependence of the magnetic susceptibility for the FeNbCuSiB alloys. The domain susceptibility decreases with increasing temperature from $1.7 \times 10^{5}$ at $20^{\circ} \mathrm{C}$ to $10^{5}$ at $180{ }^{\circ} \mathrm{C}$. This variation should be interpreted as a consequence of the anisotropy energy difference between the transforming phases and indicated the presence of a transition form.

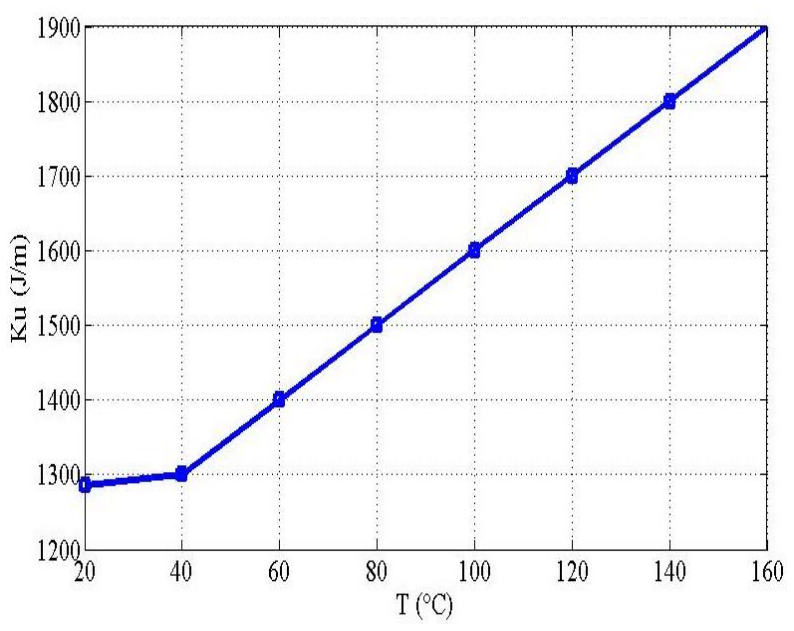

Figure 10: Variations of anisotropy energy with the temperature 
The results also show that the average domain thickness $d$ and the angle between the magnetic field and the magnetization $\alpha$ do not depend on the temperature.

\section{Conclusions}

To describe the magnetic behaviour of a linear anisotropic ferromagnetic material, a theoretical model has been presented. The mechanisms of wall displacement and coherent rotation have been taken into account in this model. An expression of the complex permeability versus the frequency has been derived. An experimental has been used to measure the open circuit and short circuit impedance of a toroidal core made from a nanocrystalline material. The variations of the complex impedance have been obtained versus the frequency and at several temperatures. The domain susceptibility and the anisotropy energy have been extracted from experimental data and using the mathematical model. Their dependence versus the temperature has been analysed. In the next future, we intend to implement the obtained results in a compact model to take into account the magnetic constraints and heat transfer in a power electronic converter.

\section{How to Cite this Article:}

H. Lagha, H. Belmabrouk, and H. Chazal, "Complex Permeability Measurements in a Nanocrystalline Toroidal Core", J. Mod. Mater., vol. 1, no. 1, pp. 2-8, Jun. 2016. doi:10.21467/jmm.1.1.2-8

\section{References}

[1] P. Szczesniak, J. Kaniewski, M. Jarnut, "AC-AC power electronic converters without DC energy storage: A review", Energ Convers Manage, vol. 92, pp. 483-497, 2015.

[2] S. S. Hong, P. A. Huff, P. C. Leach, "Chapter EightElectrical Fundamentals and Power System Principles", Pumping Station Design (Third Edition), pp. 8.1-8.41, 2008.

[3] A. Hughes, B. Drury, "Chapter two-Introduction to power electronic converters for motors drives", Electric motors and drives (Fourth Edition), pp. 39-71, 2013.

[4] V. Prasanth, P. Bauer, J.A. Ferreira, H. Polinder, "Review of analytical methods to extract magnetic parameters of an inductively coupled circuit", Emerging Technologies: Wireless Power (WoW), pp. 1-8, 2015.

[5] A. Hilal, M. A. Raulet, C. Martin, F. Sixdenier, "A Comparative Study: Dynamic and Thermal Behavior of Nanocrystalline and Powder Magnetic Materials in a Power Converter Application", Journal of Electronic Materials, vol. 44, No. 10, pp. 3768-3776, 2015.
[6] B. Ahmadi, H. Chazal, T. Waeckerle, J. Roudet, "Studying behavior of multilayer materials: A 1-D model correlated to magnetic domain walls through complex permeability", Journal of Magnetism and Magnetic Materials, vol. 320, pp. e708-e711, 2008.

[7] B. Ahmadi, "Composant magnétique intégré en alliage FeNiCrCu pour l'électronique de puissance", Ph.D. thesis, University of Grenoble, 2010.

[8] F. Mesmin, B. Ahmadi, H. Chazal, A. Kedous Lebouc, F. Sixdenier, "Improving reliability of magnetic mutual impedance measurement at high excitation level", in IEEE International Instrumentation and Measurement Technology Conference, China, pp. 1, 2011.

[9] E. Tka, K. Cherif, J. Dhahri, E. Dhahri, H. Belmabrouk, E. Hlil, "Effect of Al substitution on magnetocaloric effect in $\mathrm{La} 0.57 \mathrm{Nd} 0.1 \mathrm{Sr} 0.33 \mathrm{Mn} 1-\mathrm{xAlxO}$ polycrystalline near room temperature", J. Alloys Compounds, vol. 518, 32-37, 2012.

[10] Agilent Impedance Measurement Handbook, A guide to technology and techniques, 4th Edition. (Agilent Technologies, USA, 2009.

[11] H. Lagha, H. Chazal, H. Belmabrouk, "A New Approach for generating compact thermal models", in International Conference on Electrical Sciences and Technologies, Tunisia, pp. 1-7, 2014

[12] F. Nasri, F. Echouchene, M. F. Ben Aissa, I. Graur, and H. Belmabrouk, "Investigation of self-heating effects in a 10$\mathrm{nm}$ SOI-MOSFET with an insulator region using electrothermal modeling", IEEE Trans. Electron Devices, vol. 62, No.8, pp. 2410-2415, 2015.

[13] S. Yoon, Determination of the temperature dependence of the magnetic anisotropy constant in magnetite nanoparticles, J Korean Phys Soc., vol. 59, No. 5, pp. 3069-3073, 2011.

[14] S. Yoon, K.M. Krishnan, Temperature dependence of magnetic anisotropy constant in manganese ferrite nanoparticles at low temperature, J. Appl. Phys., vol. 109, No. 07B534, pp. 1-3, 2011.

Publish your research article in AIJR journals-

$\checkmark$ Online Submission and Tracking

$\checkmark$ Peer-Reviewed

$\checkmark$ Rapid decision

$\checkmark \quad$ Immediate Publication after acceptance

$\checkmark$ Articles freely available online

$\checkmark$ Retain full copyright of your article.

Submit your article at journals. aijr.in

Publish your books with AIJR publisher-

$\checkmark$ Publish with ISBN and DOI.

$\checkmark$ Publish Thesis/Dissertation as Monograph.

$\checkmark$ Publish Book Monograph.

$\checkmark$ Publish Edited Volume/ Book.

$\checkmark$ Publish Conference Proceedings

$\checkmark$ Retain full copyright of your books.

Submit your manuscript at books.aijr.org 